\title{
Supporting Multi-Level User-Driven Detection of Guideline Interactions
}

\author{
Luca Piovesan ${ }^{1}$, Gianpaolo Molino², Paolo Terenziani ${ }^{3}$ \\ ${ }^{1}$ Dipartimento di Informatica, Università degli Studi di Torino, Torino, Italy \\ ${ }^{2}$ Azienda Ospedaliera San Giovanni Battista, Torino, Italy \\ ${ }^{3}$ DISIT, Università del Piemonte Orientale “Amedeo Avogadro", Alessandria, Italy \\ piovesan@di.unito.it,gianpaolo.molino@virgilio.it,paolo.terenziani@unipmn.it
}

Keywords: Computer-interpretable clinical guidelines, Comorbidity treatment, Knowledge representation, Ontologies, Guideline interaction detection.

\begin{abstract}
Clinical practice guidelines are widely used to support physicians, but only on individual pathologies. The treatment of patients affected by multiple diseases (comorbid patients) requires the development of new approaches, supporting physicians in the detection of interactions between guidelines. We propose a new methodology, supporting flexible and physician-driven search and detection. In particular, we provide a flexible and interactive mechanism to navigate guidelines and our ontology of interactions (between drugs, or between actions' goals) at multiple levels of detail, focusing on specific parts of it (e.g., on a specific pair of actions, or of drugs) to look for interactions. We introduce the notion of "navigation tree", as the basic data structure to support multiple-level interaction analysis, and describe navigation and focusing algorithms operating on it. We also introduce a visualization tool that is based on the "navigation tree", and further enhances the user-friendliness of our approach.
\end{abstract}

\section{INTRODUCTION}

Clinical practice guidelines (CPGs) are defined as "systematically developed statements to assist practitioner and patient decisions about appropriate healthcare for specific clinical circumstances" (Committee to Advise the Public Health Service on Clinical Practice Guidelines, Institute of Medicine 1990).

CPGs exploitation is meant to improve the quality and to reduce the cost of healthcare, putting evidence based medicine into practice, and is progressively spreading in several countries. As a matter of fact, a lot of national and international institutions have recently been engaged in developing and disseminating CPGs. Thousands of CPGs have been devised in the last years. For instance, the Guideline International Network (Guidelines International Network n.d.) groups 100 organizations of 48 countries, and provides a library of more than 6500 CPGs. CPGs aim to reduce errors, unjustified practice variation and wasteful commitment of resources, and encourage best practices and accountability in medicine.

Moreover, the medical community has started to recognize that a computer-based management of CPGs can further increase CPG advantages, providing relevant benefits (e.g. automatic connection to the patient databases, and decision making support) to care providers and patients.

In recent years, the research about computerized guidelines has reached a relevant role within the Medical Informatics community, and many different approaches and projects have been developed to create domain-independent computer-assisted tools for managing, acquiring, representing and executing computer-interpretable clinical guidelines (henceforth CIGs). See e.g. the collections (Gordon \& Christensen 1995; Fridsma 2001; Ten Teije et al. 2008; Peleg 2013)).

By definition, clinical guidelines address specific clinical circumstances (i.e., specific diseases). However, unfortunately, specific patients may be affected by more than one disease. The treatment of patient affected by multiple diseases (comorbid patients) is one of the main challenges for the modern healthcare, also due to the aging of population, and the consequent increase of chronic 
diseases. This sets up the urgent need of developing ways of merging multiple single-disease interventions to provide professionals' assistance to comorbid patients (Riaño \& Collado 2013).

However, though some CPGs covering frequently occurring comorbidities might be devised, there is a need for formal methodologies to support physicians in the detection and resolution of interactions between guidelines, and, ultimately, in the process of merging two or more guidelines. As a result, in the very last years some computer-based approaches have started to face this problem (see the discussion in Section 5).

In this paper, we focus on one central issue in the management of multiple CIGs, namely the development of a methodology addressing interaction detection. In a recent work (Piovesan et al. 2014), we have identified three different knowledge levels at which interactions might occur: (i) level of the intentions of the CIG actions, (ii) level of the effects of the drug categories (recommended by the pharmaceutical actions in the CIGs), and (iii) level of drugs. We have also pointed out that, in turn, levels (i) and (ii) may be structured at different levels of abstraction. Indeed, though a large variety of representation formalisms exists (Ten Teije et al. 2008), most CIG formalism support a hierarchical decomposition of guidelines at multiple levels of detail, in which composite actions may be represented, and then refined (possibly at different levels of abstraction) into their components. At the finest level of detail therapeutic pharmaceutical actions in the guideline may recommend, depending on the accuracy, the use of drugs or drugs categories, or active principles (thus, also the interactions between drug categories must be considered). In turn, drug categories are usually structured in a hierarchy representing different levels of detail (see, e.g., ATC (WHO Collaborating Centre for Drug Statistics Methodology n.d.)). In (Piovesan et al. 2014), we have also proposed an ontological representation for the interactions at the different levels, as well as an algorithm that, given two actions (or drugs), automatically queries the ontology to detect interactions between them.

The main goal of this paper is that of extending the approach in (Piovesan et al. 2014) to provide user physicians with a flexible support to navigation and focusing (considering both CIGs and ontology, at the different levels of detail), in order to interactively identify actions/drugs on which interaction analysis should be performed.

\section{PROBLEMS AND METHODOLOGY}

The treatment of interactions between CIGs is a very challenging one, involving difficult problems both at the knowledge and at the process level.

At the knowledge level, two main limitations have to be faced:

(K1) defining and acquiring "a priori" a new guideline for any possible co-morbidity (i.e., for any possible combination of two or more CIGs) is not realistic in practice;

(K2) defining and acquiring, for each possible pair of CIGs G1 and G2, and for each possible pair of actions $(\mathrm{a} 1 \in \mathrm{G} 1, \mathrm{a} 2 \in \mathrm{G} 2)$ the interactions between them is practically unfeasible, too.

At the process level, an automatic process that, considering two input CIGs G1 and G2, provides as output the possible interactions between each possible pair of actions (a1€G1, a2€G2) is technically feasible, but practically useless for user physicians, since the problem is combinatorial, and too many interactions would be provided to the users (consider, in particular, the usual dimensions of realworld CIGs, and the number of alternative paths they contain).

In this paper, we propose a methodology that overcomes the above problems.

At the knowledge level, we (K1) consider CIGs developed for single diseases, and (K2) employ an ontology in which possible interactions between actions (or, better, between their goals and intentions) are modelled independently of the specific CIGs.

At the process level, we propose a mixedinitiative algorithm for the detection of interactions which, taking in input two CIGs, (i) allows user physicians to integrate their knowledge in order to focus such detection on relevant sets of actions, and (ii) exploits the (guideline independent) ontological knowledge to find and analyse the interactions between such focused parts.

Notice that the navigation and focusing phase is interactive and physician-driven, and may be facilitated by a user-friendly graphical interface. On the other hand, the interactions between the focused parts of the CIGs can be automatically provided by the system (the navigation on the ontology and the inferences on it are hidden to user-physicians). A distinguishing feature of our approach is also flexibility: it supports the navigation and selection of the focus at different levels of abstraction.

The goal of this paper is to propose a systemindependent methodology. We only assume that (i) CIGs can be structured at different levels of detail, as a hierarchical graph, (ii) CIGs contain, besides 
composite actions, also actions prescribing the administration of drug categories (or, possibly, even specific drugs). Specifically, in the following, we exemplify our approach considering the following representation (used in GLARE): each action has a type attribute, which is link to an ontological concept, and at least one aimsTo attribute, linking it to its relative intentions in the ontology. Additionally, pharmaceutical actions have a substance attribute, linking them to a drug category or to a specific drug in the ontology.

Our implementation is based on the new version of GLARE built within the GINSENG project (Terenziani et al. 2014; Terenziani et al. 2013).

\section{ONTOLOGY OF INTERACTIONS}

In (Piovesan et al. 2014), we fully detailed an ontology of interactions, which is grounded on the concepts of action goals, called intentions and of administered drugs, which represent the main sources of interactions between CIGs.

Before describing the knowledge representation, it is worth stressing that our long-term goal is to develop a decision support system highly collaborative with the user. Following this desideratum, the knowledge representation and the inferences made on it should be human-friendly enough to be understood by the user physician, as a sort of "second opinion". Indeed, in the medical field, physician cannot trust "black box" tools that simply output suggestions without explain to the user (in an "understandable" way) how such conclusions have been reached.

In our approach, action intentions are modelled as desired variations, with certain modalities (increasing, decreasing, stability), of some parameters of the patient status (attributes). Intentions are also organized along an ISA and PART-OF taxonomy.

Like intentions, drugs are organized along a taxonomy of drug categories, exhibiting, at the bottom level, specific drugs. For such hierarchy, we exploit existing classifications, such as the consensus ATC (WHO Collaborating Centre for Drug Statistics Methodology n.d.). Each drug (or drug category) is related with effects it causes, which are modelled as variations of patient's status attributes, just as the intentions.

As mentioned in the introduction, interactions may occur at each level of abstraction (i.e., each level of the two taxonomies), and the ontology supports the representation of interactions at all the levels.

An Intention interaction is an interaction between two intentions, and it is described by a type. We identified three basic types: independence (intentions do not interact), concordance (intentions reinforce each other), and discordance (intentions negatively interact with each other). However, more "sharp" types of interactions can also be added. For instance, the opposite type (as subtype of discordance) could be added to cope with intentions focusing on the same attribute, with opposite modalities.

Drug interactions occur between two drugs or drug categories. A drug interaction is characterized by the modality of the variation that it causes in an effect of the two drugs it involves.

Both types of interactions can be annotated by links to the literature showing their evidence.

It is worth stressing that the interactions we model are action and guideline independent because they involve (action) intentions and drugs, which are general concepts. Thus, differently from some other approaches, when modelling a new CIG it is not needed to specify all the interactions between the new CIG and the existing ones because they are autonomously recognized following the relations between the actions and their intentions and drugs prescribed (for pharmaceutical ones).

In the following, Figure 1 shows a glimpse of part of the interaction ontology, focusing on the modelling of interactions. At the moment, the ontology has been validate using parts of guidelines (see examples in this paper and in (Piovesan et al. 2014)). However, it is scheduled to be integrated in METAGLARE (Terenziani et al. 2014), recently developed.

\section{A FLEXIBLE USER-DRIVEN ALGORITHM FOR INTERACTION DETECTION AT MULTIPLE LEVELS OF ABSTRACTION}

\subsection{Background}

In this section, we describe our mixed-initiative approach to the interaction detection between CIGs. It is worth stressing that our main goal is to integrate three fundamental knowledge sources:

(i) Knowledge deriving from the CIGs structure, such as the decomposition of high level actions in lower level ones, 
and the sequence of actions to be executed

(ii) Knowledge about intentions, drugs, and their interactions. We detailed how we organized such a basic medical knowledge in the previous section

(iii) User physician knowledge about the context and relevant parts of CIGs for the specific case

In particular, given the high number of alternative paths and actions for each CIGs, the physician's knowledge regarding the context is of fundamental importance. Indeed, without considering such an information, the output of an autonomous tool could be not useful for the user: it would contain too many non-organized information, and most of them would be irrelevant for the specific case. Exploiting out tool, the user physician can a priori discard uninteresting parts of CIGs and focus only on the relevant ones, obtaining, in addition, a well-structured, easy-to-use and easy-to-analyse output. In order to accomplish such a result, we aim at devising a flexible and interactive detection tool allowing physicians to navigate through the different abstraction levels, thus supporting the natural methodology they adopt to cope with CIG analysis. For instance, at the highest level, a physician may want to start to consider only the interactions between the intentions of the high-level actions of the guidelines. Then, focusing on a specific part of the guideline, (s)he may want to move down to a more detailed analysis, considering the decomposition of composite action into its parts, and/or the specific drugs category considered in order to reach the high-level intentions. In general, our approach will provide physicians with the possibility of moving in both directions, i.e., going down from a general to a more specific analysis, or moving up, from a specific analysis to a higher level of abstraction.

Another important contribution of our approach is the possibility of extending CIGs with the knowledge in the ontology. For instance, many guidelines only recommend drug categories. However, at the very end, a specific drug of that category must be prescribed to the specific patient, but there are many cases in which, while two drug categories do not interact, specific drugs of the two categories do interact. Such interactions can only be detected in case the CIG knowledge is expanded with the knowledge in the ontology.

\subsection{Data Structures}

To provide a flexible support, our algorithm must rely on suitable data structures. In particular, such structures should support the following desiderata

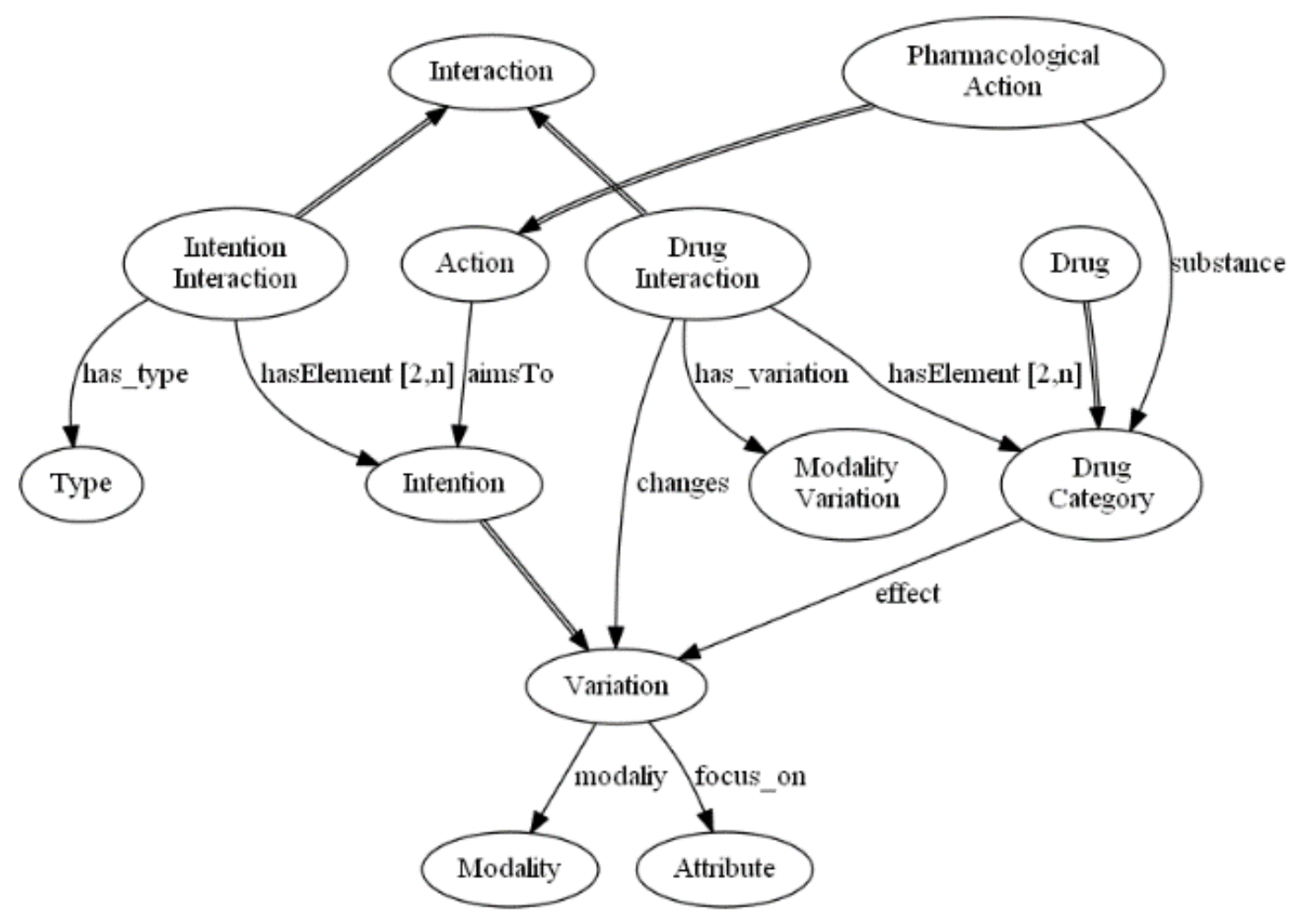

Figure 1 Ontology of Intentions, Drugs and Interactions between them. 
(i) Maintain the history of the focusing process, supporting both the addition of new CIG focuses, and the rollback to upper focuses

(ii) Maintain the association between CIG focuses and interactions

As regards specifically the representation of interactions, the data structure must also

(iii) Support the fact that interactions occur between pair of actions in the two CIG focuses

(iv) Support the fact that multiple interactions may occur between each pair of actions (since actions may have multiple intentions, and drugs may have multiple effects)

To store the history, a tree structure is adopted. Each node of the tree represents a "status" of the analysis. It consists of three elements: (i) the focused part of the first CPG (at the chosen abstraction level), (ii), the focused part of the second CPG (at the chosen abstraction level), and (iii) the list of action interactions (found at the chosen level). In turn, each "focused part" of a CPG simply consists of a selection of nodes from the CPG itself.

Actions interactions derive from intention or drug interactions. In particular, the list contains (when filled by the algorithm) an interaction of two actions $a 1$ and $a 2$ for each pair of intentions $i 1$ (related to al) and $i 2$ (related to a2) that interact. In addition, if $a 1$ and $a 2$ are pharmaceutical actions, the list contains an interaction for each pair of drugs (or drug categories) $d l$ (substance of $a l$ ) and $d 2$ (substance of $a 2$ ) that interact. The list maintains the link to the ontological concepts, in order to allow the user to examine the reasons of such conclusions.

Initially, the root of the tree contains the two input CIGs (at the highest abstraction level) and the list of interactions is empty. Then, the tree structure expands to explicitly model (the results of) the operations performed in a session of work (see, e.g., the graphical representation in Figures 2 and 3).

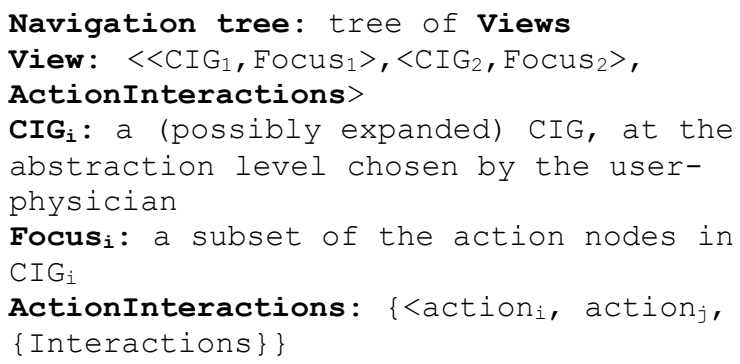

\subsection{The Interaction Detection Tool}

Our INTERACTION-DETECTION algorithms support user-driven navigation over a navigation tree, starting from a given node of the tree (i.e., from the current view).

At the beginning of a session of work, considering two guidelines $\mathrm{CIG}_{1}$ and $\mathrm{CIG}_{2}$, the tree is initialized with just the root node, consisting of the view $\left\langle\left\langle\mathrm{CIG}_{1},\{\}\right\rangle,\left\langle\mathrm{CIG}_{2},\{\}\right\rangle\right.$, \{\}$\rangle$ (i.e., at the beginning, no focuses and no interactions are identified), which is set as the current view.

At each step, the algorithm allows the user to choose between four alternative actions: STOP_ANALYSIS (which simply closes the session of work), REFINE, ROLL-UP, and DETECT.

REFINE add a new view to the navigation tree (a child of the current view), which becomes the new current view. Such a new view is initialized as a copy of the current view, but it is then refined by refining actions and/or identifying focuses, through the Zoom\&Select procedure (see below). On the other hand, ROLL-UP moves up along the navigation tree, setting the mother of the current view as the new current view. Finally, DETECT add the interactions between the focused actions to the current view. The DETECT operation exploits the links between the guideline action description and the ontology and navigates the ontology in order to find the modality of the interactions. A full description of such an operation is reported in (Piovesan et al. 2014).

Through the Zoom\&Select procedure, users can iteratively refine a view, by changing the focuses and/or expanding some of the actions they contain. Four options are possible. STOP_FOCUSING simply ends up the procedure. ADD_TO_FOCUS add some actions in a CIG into its focus, while REMOVE_FROM_FOCUS remove actions from the focuses. EXPAND supports the expansion of some of the actions in the focuses. First, the user chooses the actions to expand (variable actions_to_refine) then, while actions_to_refine is not empty, each action $a$ in it is independently refined. The REFINEMENT operation takes in input a view $(v)$ and an action $a$ in it, and performs one step of refinement. The way in which such a refinement is obtained depend on the type of $a$. If $a$ is a composite action in $v$, its expansion is simply the sub-guideline constituted by the actions composing it. On the other hand, if $a$ is a pharmaceutical action, the representation of $a$ contains the attribute substance, whose value is a link to the ontological entity representing drug category to be administered (say $\operatorname{DrugCat}_{x}$ ). Thus, $a$ is expanded as a new piece of guideline, consisting of several alternative 
pharmacological actions, one for each one of the direct descendants of DrugCat in the ontology.

The REPLACE operation simply substitutes $a$ with the newly identified refinements into $v$.

Whenever a new refinement is added, we allow users to update actions_to_refine, adding actions of the new refinement to it. In such a way, we provide users with the possibility of going on with this process until the desired level of detail is reached.

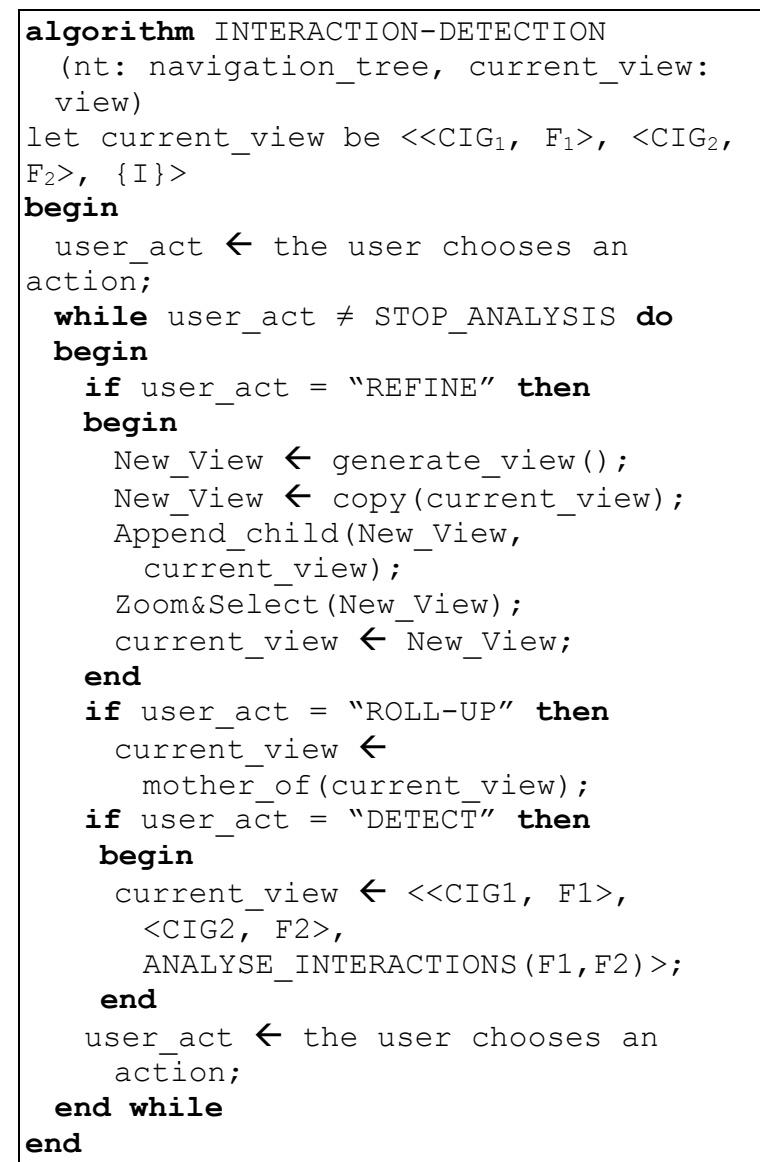

\subsection{Graphical Interface}

In the following, we explain how we integrated our algorithms with a graphical interface, in order to support users in the detection and analysis of interactions at different levels of detail. The graphical interface provides a user-friendly interaction to physicians. In Figures 2 and 3, we show how the navigation tree is displayed by the graphical interface, considering as an example a session of work. In particular, we compare a simplified part of a CIG for the postoperative management (PM), with a simplified part of a CIG for the treatment of acute otitis media (AOM).

Each node in the navigation tree is represented by three boxes: the first two show the guideline views at the respective status of expansion, while the third box contains the interactions detected between actions contained in the views, when available.

At the beginning of the detection, the interface only contains the root of the tree, with the guideline views at the highest level of abstraction.

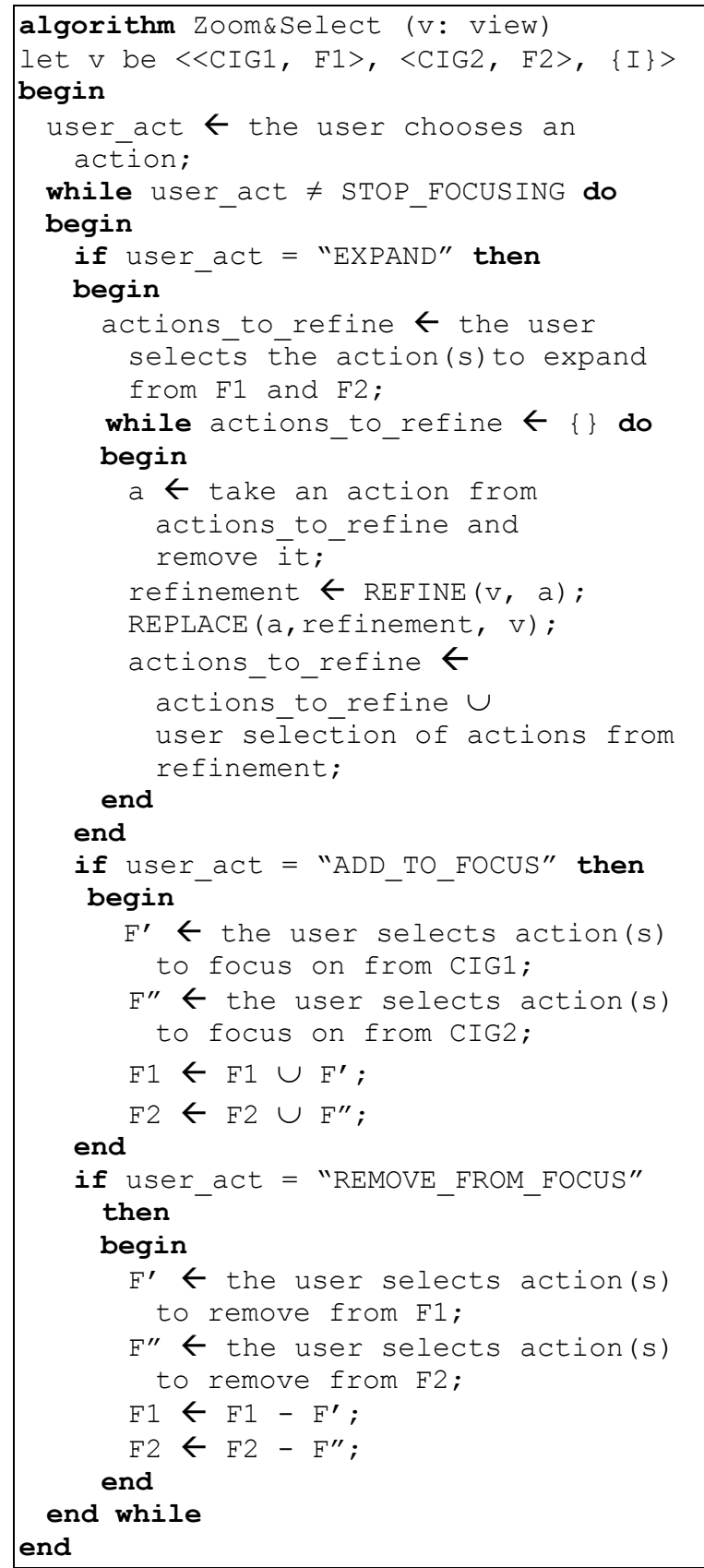




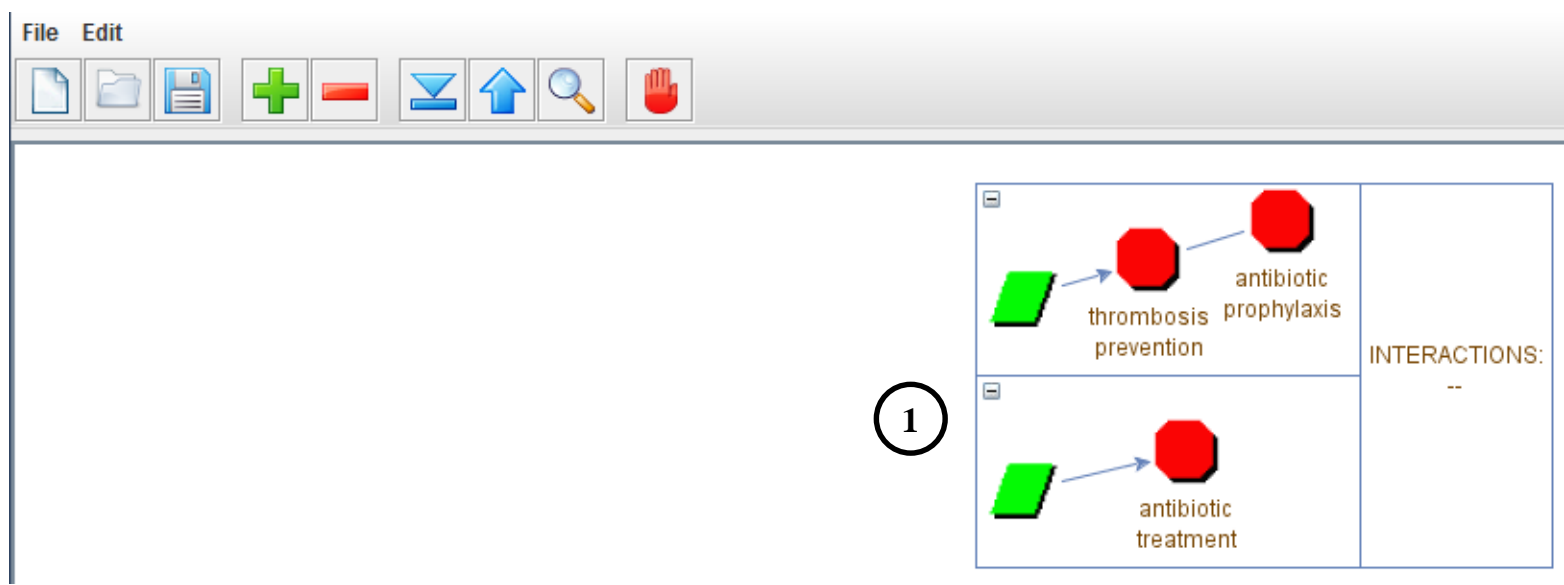

Figure 2 Graphical interface for the INTERACTION DETECTION process. Each node of the tree represents a level of expansion of the guidelines and may contains the detected interactions. The simulation continues in Figure 3.

For the sake of simplicity, in our example we suppose that the initial situation (with the guidelines at the highest level of abstraction) is represented as shown in Figure 2.

In such a situation (part (1)), the physician may want to compare the composite action "thrombosis prevention" of the PM CIG with the "antibiotic treatment" of the AOM one. In that case, (s)he selects to refine the current node. The Zoom\&Select procedure window, not shown in this paper for the sake of brevity, allows her/him to add the two actions to the focus and expand them. We briefly explain how it works. First, considering the PM CIG, the physician applies the "ADD TO FOCUS" and "EXPAND" procedures to the "thrombosis prevention" action. The result is the expansion of such action. Among the other actions, the composite action "thrombosis prevention" recommends the administration of an antithrombotic agent. Then, the physician decides to focus on such action, and in particular, among the available antithrombotic agents, on the administration of a Vitamin $\mathrm{K}$ antagonist (repeating the procedures "ADD TO FOCUS" and "EXPAND"). Now, supposing that the guideline does not specify the level of specific drugs, such expansion cannot be performed using the CIG knowledge only. Then, the system uses the knowledge in the ATC classification in order to build the expansion of the selected action, returning a decision between the administrations of all the possible Vitamin $\mathrm{K}$ antagonists (e.g., dicoumarol, warfarin, etc.), which is the expansion shown in Figure 3. At this point, the physician has reached the desired level of abstraction and a similar procedure is performed to expand the "antibiotic treatment" node for the AOM CIG. When the refinement is complete, the physician selects the action STOP_FOCUSING and the node on the left part of Figure 3 is created.

In the part (2) of Figure 3, we can see a simplified expansion of the two selected actions. Notice that the current expansion has been added to the navigation tree as a child of the first one. At this time, the third box of the node does not contain any interaction. If the user performs a DETECT action, interactions between the focused actions in the two expansions of the guidelines are automatically detected navigating the ontology and inserted in the third box of the node (part (3) of Figure 3). An interaction is detected between the administrations of warfarin and erythromycin, which causes an increase in the anticoagulant effect of the warfarin. Now, we suppose that the physician is satisfied in the exploration of this direction, and decide to explore other possible interactions: (s)he perform a ROLL-UP action, in order to set the root of the tree as the current view (the node in 2 is however maintained; see the part (4) in Figure 3). In the example, we suppose that the user selects for the new refinement the same action ("antibiotic treatment") for the AOM guideline, but another one, "antibiotic prophylaxis" for the PM one.

The Zoom\&Select procedure at this time produces the node in right part (5) of Figure 3, and the navigation can go on until the desired level of detail is reached, and the actions to be focused on are decided. At this point, DETECT can be invoked again, and so on. Obviously, the navigation tree can expand at any depth, and there is no limit for the possible alternative branches at any level, since (in principle) all alternative expansions, at all levels, can be explored and maintained in the navigation tree. 


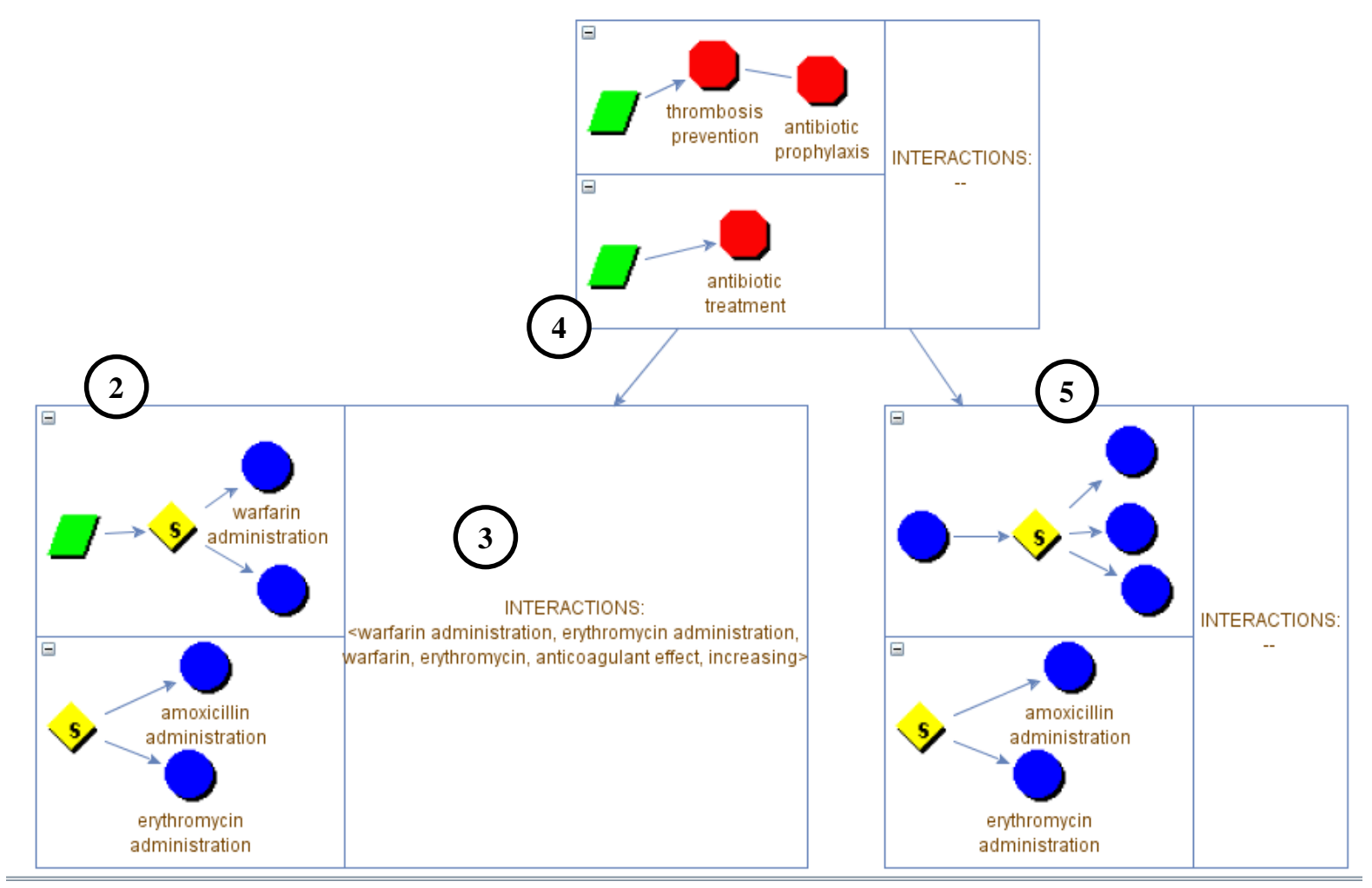

Figure 3 Second part of the simulation.

\section{RELATED WORK AND DISCUSSION}

The development of software tools and methodologies to support interaction detection is gaining an increasing attention in the last years. For instance, several current tools provide online access to pharmacological databases (Robert Wood Johnson Foundation and Partnership for Solutions 2004), or alerting systems that detect and inform about interactions (Medscape, Drugs.com, etc.). However, such tools mostly focus on drug-drug interactions only. Unfortunately, this is only a very limited support, when a physician has to detect and analyse the interactions between two or more guidelines.

To overcome such a limitation, several research approaches have been proposed in the very last years. As regards ontologies, GLINDA (Musen et al. 2011) proposes a wide ontology of cross-guideline interactions.

On the other hand, several other approaches have focused their attention on methodologies to "merge" two or more CIGs, "solving" their interactions.
Sánchez-Garzón (Sánchez-Garzón et al. 2013), for example, attempts to capture the collaborative aspect of the merging: each guideline is considered as a physician expert in the treatment of a single disease, and represented by an agent with hierarchical planning capabilities. The result is obtained through the coordination of all the agents, and respects the recommendations of each guideline. Another interesting approach, presented in (Michalowski et al. 2013) and (Wilk et al. 2013), uses constraint logic programming to identify and address adverse interactions. In this solution, a constraint logic programming (CLP) model is derived from the combination of logical models that represent each CIG, then a mitigation algorithm is applied to detect and mitigate interactions. Among rule-based systems, (López-Vallverdú et al. 2013) represents guidelines as sets of clinical actions that are modelled into an ontology. To combine two treatments, first they are unified in a unique treatment, then a set of "combination rules" is applied to detect and avoid possible interactions. A model-based combination of CIGs is purposed in (Riaño \& Collado 2013), in which guidelines expressed in a particular formalism can be automatically merged through a combining operator. 
Jafarpour (2013) uses semantic-web rules and an ontology for the merging criteria. Given these, an Execution Engine dynamically merges several CIGs according to merge criteria.

On the other hand, the approach in this paper focuses on interaction detection, rather than on guideline merge. In this sense, our approach is largely complementary with respect to the above approaches in the literature, and can be integrated with them. However, two main advantages of the approach in this paper are (i) the fact that it is flexible, interactive and user-driven: instead or proposing "black-box solutions" to physicians, we aim at providing them with user-friendly investigation and decision supports; (2) the fact that is allows the analysis at different levels of detail.

The approach in this paper is based on (Piovesan et al., 2014). Indeed, as discussed in Section 2, an automatic process that provides as output the possible interactions between each possible pair of actions between two CIGs is practically useless for user-physicians, since the problem is combinatorial, and too many interactions would be provided as output. Thus, we suggest to split interaction analysis into two phases, which can be iteratively repeated in an interactive and physician-driven process: (1) focus on specific actions/drugs (at a specific level of detail), and (2) detect interactions on them. While the work in (Piovesan et al., 2014) mainly focuses on the second phase, in this paper we extend it to cope with the first one. To achieve such a goal, this paper presents three major original contributions: (1) analysis of the requirements for the data structures, and their definition (see the navigation tree, in Section 4.2); (2) definition of a flexible and interactive focusing algorithm (Section 4.3); (3) definition of a user-friendly graphical interface (Section 4.4). The main limitation of the current approach is, in our opinion, the fact that it has only undergone a limited experimental evaluation. Up to now, it has been tested only on simplified guidelines or part of them, such as the ones described in the Section 4, by two physicians of Azienda Ospedaliera San Giovanni Battista ("Molinette" Hospital) in Turin. Though the test has been quite successful, a more systematic and intensive experimental evaluation should be required, and this is the goal of our future work.

\section{CONCLUSIONS}

The treatment of patients affected by multiple diseases (comorbid patients) is one of the main challenges for the modern healthcare, also due to the aging of population, and to the increase of chronic diseases. Recent studies demonstrates that various types of interactions must be taken into account when merging two (or more) CIGs, and propose and ontology of interactions (Piovesan et al. 2014; Musen et al. 2011). However, to the best of our knowledge, our approach is the first one that, having identified different levels of abstractions in the analysis of interactions, supports user-driven and interactive interaction detection over them.

Our flexible approach to interaction detection, operating at different levels of abstractions, may support expert physicians to analyse "abstractly" (i.e., just considering the CIGs, with no reference to a specific patient) the interactions between two or more CIGs that are commonly used together (e.g., to provide some "partial merge" between them). In such a case, the possibility of reasoning about "highlevel" actions is certainly crucial. Moreover, our approach can also support a physician treating a specific comorbid patient. In such a context, though the abstraction facilities are certainly helpful, the possibility of moving from the "general" actions in the guidelines to study of the interactions of specific drug categories (and drugs) can play a crucial role.

In our short-term future work, we aim at proposing a more extensive experimental evaluation of the current approach, and at extending it to cope also with "patient-guideline action" interactions and "patient-drug" interactions, and with the temporal issues (e.g., not all interactions between CIGs are possible, due to the temporal constraints between guideline actions). In our long-term future work, we will support physicians also in the interaction solving, and, finally, in merging multiple guidelines in the treatment of a specific patient.

\section{ACKNOWLEDGMENTS}

The work described in paper was partially supported by Compagnia di San Paolo, in the Ginseng project.

\section{REFERENCES}

Committee to Advise the Public Health Service on Clinical Practice Guidelines, Institute of Medicine, 1990. Clinical practice guidelines directions for a new program M. J. Field \& K. N. Lohr, eds., Washington, D.C.: National Academy Press. Available at: http://site.ebrary.com/id/10068353 [Accessed July 28, 2014]. 
Fridsma, D.B., 2001. Special Issue on Workflow Management and Clinical Guidelines. Journal of the American Medical Informatics Association, 22(1), pp.1-80.

Gordon, C. \& Christensen, J.P. eds., 1995. Health telematics for clinical guidelines and protocols, Amsterdam, Netherlands: IOS Press.

Guidelines International Network, Guidelines International Network website. Available at: http://www.g-i-n.net/ [Accessed October 14, 2014].

Jafarpour, B. \& Abidi, S.S.R., 2013. Merging Disease-Specific Clinical Guidelines to Handle Comorbidities in a Clinical Decision Support Setting. In AIME. pp. 28-32.

López-Vallverdú, J.A., Riaño, D. \& Collado, A., 2013. Rule-Based Combination of Comorbid Treatments for Chronic Diseases Applied to Hypertension, Diabetes Mellitus and Heart Failure. In R. Lenz et al., eds. Process Support and Knowledge Representation in Health Care. Lecture Notes in Computer Science. Springer Berlin Heidelberg, pp. 30-41.

Michalowski, M. et al., 2013. Using Constraint Logic Programming to Implement Iterative Actions and Numerical Measures during Mitigation of Concurrently Applied Clinical Practice Guidelines. In N. Peek, R. M. Morales, \& M. Peleg, eds. Artificial Intelligence in Medicine. Lecture Notes in Computer Science. Springer Berlin Heidelberg, pp. 17-22.

Musen, M.A. et al., 2011. GLINDA Interaction Ontology. Available at: http://glindaproject.stanford.edu/guidelineinteractionontology .html [Accessed October 17, 2014].

Peleg, M., 2013. Computer-interpretable clinical guidelines: A methodological review. Journal of Biomedical Informatics, 46(4), pp.744-763.

Piovesan, L., Molino, G. \& Terenziani, P., 2014. An ontological knowledge and multiple abstraction level decision support system in healthcare. Decision Analytics, 1(1). Available at: http://www.decisionanalyticsjournal.com/content $/ 1 / 1 / 8$.

Riaño, D. \& Collado, A., 2013. Model-Based Combination of Treatments for the Management of Chronic Comorbid Patients. In Artificial Intelligence in Medicine. 14th Conference on Artificial Intelligence in Medicine. Springer, pp. 11-16.

Robert Wood Johnson Foundation and Partnership for Solutions, 2004. Chronic Conditions: Making the Case for Ongoing Care. Available at: Chronic
Conditions: Making the Case for Ongoing Care [Accessed October 19, 2014].

Sánchez-Garzón, I. et al., 2013. A Multi-agent Planning Approach for the Generation of Personalized Treatment Plans of Comorbid Patients. In N. Peek, R. Marín Morales, \& M. Peleg, eds. Artificial Intelligence in Medicine. Berlin, Heidelberg: Springer Berlin Heidelberg, pp. 23-27. Available at: http://link.springer.com/10.1007/978-3-64238326-7_4 [Accessed October 17, 2014].

Ten Teije, A., Miksch, S. \& Lucas, P. eds., 2008. Computer-based medical guidelines and protocols: a primer and current trends, Amsterdam, Netherlands: IOS Press.

Terenziani, P. et al., 2013. Towards a Second Generation of Computer Interpretable Guidelines. In M. Helfert, C. Francalanci, \& J. Filipe, eds. DATA 2013 - Proceedings of the 2nd International Conference on Data Technologies and Applications, Reykjavík, Iceland, 29 - 31 July, 2013. SciTePress, pp. 199-205.

Terenziani, P., Bottrighi, A. \& Rubrichi, S., 2014. META-GLARE: a meta-system for defining your own CIG system: Architecture and Acquisition. In 6th International Workshop on Knowledge Representation for Health Care. pp. 92-107.

WHO Collaborating Centre for Drug Statistics Methodology, Anatomical Therapeutic Chemical classification system. Available at: http://www.whocc.no/atc/ [Accessed October 14, 2014].

Wilk, S. et al., 2013. Mitigation of adverse interactions in pairs of clinical practice guidelines using constraint logic programming. Journal of biomedical informatics, 46(2), pp.341-353. 